\title{
Overexpression of squamous cell carcinoma antigen in idiopathic pulmonary fibrosis: clinicopathological correlations
}

\author{
F Calabrese, ${ }^{1}$ F Lunardi, ${ }^{1}$ C Giacometti, ${ }^{1}$ G Marulli, ${ }^{2}$ M Gnoato, ${ }^{3}$ P Pontisso, ${ }^{3}$ M Saetta, ${ }^{2}$ \\ M Valente, ${ }^{1}$ F Rea, ${ }^{2}$ E Perissinotto, ${ }^{4}$ C Agostini ${ }^{3}$
}

${ }^{1}$ Department of Diagnostic Medical Sciences and Special Therapies, University of Padua Medical School, Padua, Italy;

${ }^{2}$ Department of Cardiothoracic Sciences, University of Padua Medical School, Padua, Italy:

${ }^{3}$ Department of Clinical and

Experimental Medicine,

University of Padua Medical

School, Padua, Italy:

${ }^{4}$ Department of Environmental Medicine and Public Health, University of Padua Medical School, Padua, Italy

Correspondence to:

Professor F Calabrese

Department of Diagnostic

Medical Sciences and Special

Therapies, University of Padua

Medical School, Via Gabelli, 61

Padua, Italy; fiorella.calabrese@ unipd.it

Received 9 August 2007

Accepted 4 February 2008

Published Online First

14 March 2008

\begin{abstract}
Background: Idiopathic pulmonary fibrosis (IPF) is a chronic progressive disorder with a poor prognosis. Epithelial instability is a crucial step in the development and progression of the disease, including neoplastic transformation. Few tissue markers for epithelial instability have been reported in IPF. Squamous cell carcinoma antigen (SCCA) is a serine protease inhibitor typically expressed by dysplastic and neoplastic cells of epithelial origin, more often in squamous cell tumours. At present, no information is available on its expression in IPF.

Methods: SCCA and transforming growth factor $\beta$ (TGF $\beta$ ) expression in surgical lung biopsies from 22 patients with IPF and 20 control cases was examined. An in vitro study using A549 pneumocytes was also conducted to investigate the relationship between SCCA and TGF $\beta$ expression. SCCA and TGF $\beta$ epithelial expression was evaluated by immunohistochemistry and reverse transcription-PCR (RT-PCR). SCCA values were correlated with different pathological and clinical parameters. Time course analysis of TGF $\beta$ expression in A549 pneumocytes incubated with different SCCA concentrations was assessed by real time RT-PCR.
\end{abstract}

Results: SCCA was expressed in many metaplastic alveolar epithelial cells in all IPF cases with a mean value of $24.9 \%$ while it was seen in only two control patients in up to $5 \%$ of metaplastic cells. In patients with IPF, SCCA correlated positively with extension of fibroblastic foc $(r=0.49, p=0.02)$, expression of TGF $\beta(r=0.78$, $p<0.0001)$ and with carbon monoxide transfer factor decline after 9 months of follow-up $(r=0.59, p=0.01)$. In vitro experiments showed that incubation of cultured cells with SCCA induced TGF $\beta$ expression, with a peak at $24 \mathrm{~h}$.

Conclusion: Our findings provide for the first time a potential mechanism by which SCCA secreted from metaplastic epithelial cells may exert a profibrotic effect in IPF. SCCA could be an important biomarker in this incurable disease.

Idiopathic pulmonary fibrosis (IPF), morphologically characterised by an usual interstitial pneumonia (UIP), represents a progressive disease of unknown aetiology that continues to be associated with high morbidity and mortality. ${ }^{1}$

The key pathological features of this disorder include epithelial damage/activation, fibroblastic/ myofibroblastic foci formation and extracellular matrix remodelling. ${ }^{2}$ Although previous studies on fibrogenesis have focused on the role of inflammation in favouring fibroblast activation and fibrosis, the current paradigm suggests the pivotal role of epithelial deregulation in the disease pathogenesis. $^{23}$ Epithelial injury and subsequent deregulated repair result in cytokine release, excess of extracellular matrix deposition and abnormal mesenchymal cell activation and proliferation. Among profibrogenetic cytokines, transforming growth factor $\beta$ (TGF $\beta$ ) represents the key growth factor which leads to mesenchymal differentiation, myofibroblast infiltration and proliferation. As a consequence, the areas of immature fibrosis are progressively invaded by small aggregates of actively proliferating myofibroblasts and fibroblasts termed fibroblastic foci (FF) whose number and extension have been associated with the prognosis of the disease. ${ }^{4}$

Epithelial instability has been largely documented by the presence of frequent evidence of morphological changes through variegated cellular alterations, such as hyperplasia, atypia, squamous metaplasia, dysplasia and eventually carcinoma. ${ }^{5-9}$ The frequent occurrence of lung carcinoma, particularly squamous cell type, in patients with IPF/ UIP is one of the contributing factors to a poor prognosis. ${ }^{10} \mathrm{~A}$ few tissue markers for epithelial instability have recently been described as being overexpressed in lung tissue from patients with IPF/UIP, including the expression of the K-ras gene with point mutation and the presence of multiple mutations of $\mathrm{p} 53$, which have been detected in type II alveolar pneumocytes of IPF/UIP lungs. ${ }^{11}$ Also, overexpression of wild-type p53 has been reported by several authors. Its upregulation in the face of chronic DNA damage may increase the potential p53 mutation leading to an imbalance of different growth factors and consequently increased tumorigenesis in IPF. ${ }^{12}{ }^{13}$ A more frequent occurrence of guanine nitration has recently been found in metaplastic and neoplastic cells from patients with IPF/UIP, indicating that nitrative stress is an important step in epithelial damage at risk of neoplastic transformation. ${ }^{14}$

Squamous cell carcinoma antigen (SCCA) is a serine protease inhibitor typically expressed by dysplastic/neoplastic cells of epithelial origin, including lung cancer. ${ }^{15}$ Recent studies indicate that both SCCA1 and SCCA2, the two isoforms so far identified, ${ }^{16}$ protect neoplastic cells from apoptotic death induced by several types of stimuli, and in vivo experiments demonstrate that SCCA can promote tumour growth. ${ }^{17}$ The main goal of the present study was to assess expression of SCCA both as mRNA and protein in lung biopsies from patients with IPF/UIP and from controls. To 
analyse the pathogenetic and prognostic value of SCCA, the association between its value and different clinical and pathological data, including TGF $\beta$ tissue expression, was studied. An in vitro study was also conducted to investigate the biological activity of SCCA on TGF $\beta$ expression.

\section{MATERIALS AND METHODS}

\section{Patients}

The study was performed on lung samples from 22 patients with IPF consecutively evaluated from June 2003 to August 2006 at our centre and on 20 samples from non-IPF subjects. The diagnosis of IPF was based on the diagnostic criteria of the American Thoracic Society/European Respiratory Society Consensus Classification System. ${ }^{18}$ Samples from patients with IPF were obtained from video assisted thoracoscopic lung biopsies. Written informed consent was obtained from each patient and the work was approved by the Institutional Ethics Committee. Histological examination revealed all of the major features of UIP, which is a prerequisite for the diagnosis of IPF. Mean age of the patients was 60.2 years (range 44-69); 17 of the patients were males and five were females. All patients underwent routine pulmonary function testing, including spirometry, lung volume measurement, measurement of carbon monoxide transfer factor (TlCO), arterial blood gases at rest and

Table 1 Clinical and pathological characteristics of the study population (22 patients with usual interstitial pneumonia)

\begin{tabular}{|c|c|}
\hline Characteristic & \\
\hline $\operatorname{Sex}(M: F)(n)$ & $17: 5$ \\
\hline Status (dead/alive) (n) & $2: 20$ \\
\hline Age at biopsy (y) & $60.2(6.2)(44-69)$ \\
\hline Dust exposure (yes/no) (n) & $9: 13$ \\
\hline Smoker (yes/no) (n) & $12: 10$ \\
\hline Smoking (pack-years) & $23.3(19.1)(0.45-64)$ \\
\hline Follow-up (months) (median (range)) & $9(6-11)$ \\
\hline \multicolumn{2}{|l|}{ Pathological features } \\
\hline \multicolumn{2}{|l|}{ Fibroblastic foci score (\%) } \\
\hline 1 & 4.6 \\
\hline 2 & 40.9 \\
\hline 3 & 13.6 \\
\hline 4 & 22.7 \\
\hline 5 & 4.6 \\
\hline 6 & 13.6 \\
\hline Fibroblastic foci $\left(A_{F F} \%\right)$ (median (range)) & $8.4(4.3-21.8)$ \\
\hline Fibrosis extension $\left(A_{\text {Fib }} \%\right)$ & $35.6(7.4)(22-51.8)$ \\
\hline \multicolumn{2}{|l|}{ Inflammation score $(\%)$} \\
\hline 1 & 53.8 \\
\hline 2 & 38.5 \\
\hline 3 & 7.7 \\
\hline Inflammation $\left(A_{I C} \%\right)$ & $3.0(1.4)(1.2-6.6)$ \\
\hline \multicolumn{2}{|l|}{ Spirometry at time of diagnosis } \\
\hline TIco (\% predicted) & 53.7 (12.7) (23-77) \\
\hline FEV1 (\% predicted) & 72.9 (15.7) (42-109) \\
\hline FVC (\% predicted) & 69.5 (13.6) (49-97) \\
\hline VC (\% predicted) & $71.1(12.7)(47-94)$ \\
\hline TLC (\% predicted) & 67.1 (12.9) (48-91) \\
\hline RV (\% predicted) & $67.7(25.5)(31-125)$ \\
\hline
\end{tabular}

Values are mean (SD) (range) unless otherwise stated. For normally distributed quantitative variables, values are mean (SD) (range); for not normally distributed quantitative variables, median range (IOR) are shown; for categorical variables, percentage distributions are shown.

$A_{F F}$, fibroblastic foci area; $A_{F i b}$, fibrotic area; $A_{I C}$, inflammatory cells area; $F E V_{1}$, forced expiratory volume in $1 \mathrm{~s}$; FVC, forced vital capacity; RV, residual volume; TLC, total lung capacity; TIco, carbon monoxide transfer factor; VC, vital capacity. after exercise, chest radiography and high resolution CT. The main characteristics of the subjects are shown in table 1.

Lung function data were recorded less than 6 weeks before biopsies in all IPF cases and re-evaluated after a median period of 9 months (range 6-11). At follow-up, clinical data were completed for 18 patients, two patients died before undergoing the second pulmonary function test and spirometry results were not evaluable for two cases. Data were expressed as percentage of values predicted from the subject's age, sex and height.

Fifteen patients underwent biopsies at two different sites (upper and lower lobes), and the other seven patients were biopsied at three separate sites (upper, middle and lower lobes), giving a total number of 51 biopsies. The majority of the patients were treated after biopsy with a high dose of steroids alone or in combination with azathioprine. Control lungs were obtained from non-implanted donor lungs (10 cases) and from other forms of interstitial lung diseases (ILDs, 10 cases): two non-specific interstitial pneumonias, one desquamative interstitial pneumonia, one Langerhans-cell histiocytosis, one lymphangioleiomyomatosis, two respiratory bronchiolitis interstitial lung diseases, one cryptogenic organising pneumonia, one mixed pneumoconiosis and one hypersensitivity pneumonitis. The donors (five males and five females, mean age 30 (17) years, all no smokers) died of cerebral trauma and stayed less than 2 days in intensive care without evidence of lung infections or other complications. Patients affected by other forms of ILDs (three males and seven females; mean age 45 (15) years, all smokers except patients with lymphangioleiomyomatosis and hypersensitivity pneumonitis) were defined by the presence of clinical, radiological and histological evidence of specific ILD. All lung tissues were formalin fixed and paraffin embedded following standard protocols.

\section{Histology and morphometry}

Fibroblastic foci (FF) and inflammatory cells (IC) were evaluated using a semiquantitative method. In particular, FF were analysed using a Brompton score $e^{4}$ and IC was scored as follows: less than $10 \%$ of lung tissue examined (score 1 ), more than $10 \%$ and less than $30 \%$ of lung tissue examined (score 2) and more than $30 \%$ of lung tissue examined (score 3 ). In all samples from each patient, the extent of fibrosis, inflammation and FF were also measured by computerised morphometric analyses (Image Pro-plus V.5). The extent of fibrosis was quantified on lung sections stained by Azan-Mallory, as previously described. ${ }^{19} \mathrm{FF}$, IC and fibrosis were analysed on 10 random fields in the same section of imaged lesions at 50-fold magnification, excluding the areas of honeycombing. In each selected field, the ratio of FF, IC and fibrotic areas $\left(A_{F F}, A_{I C}\right.$, $\mathrm{A}_{\mathrm{FIB}}$ ) were calculated, dividing the total $\mathrm{A}_{\mathrm{FF}}, \mathrm{A}_{\mathrm{IC}}$ and $\mathrm{A}_{\mathrm{FIB}}$ by the

Table 2 Oligonucleotide sequences of primers used to amplify GAPDH (housekeeping), TGF $\beta$ and SCCA

\begin{tabular}{llll}
\hline Primer & Sequence 5'-3' & $\begin{array}{l}\text { Annealing } \\
\text { temp (' } \mathbf{C})\end{array}$ & $\begin{array}{l}\text { Amplicon size } \\
\text { (bp) }\end{array}$ \\
\hline GAPDH Fw & GGGCTCTCCAGAACATCATCC & 60 & $130 \mathrm{bp}$ \\
GAPDH Rv & GTCCACCACTGACACGTTGG & & \\
SCCA Fw & GGCAGCTGCAGCTTCTG & 55 & $80 \mathrm{bp}$ \\
SCCA Rv & AGCCGCGGTCTCGTGC & & \\
TGF $\beta$ Fw & GCCCTGGACACCAACTATTGC & 60 & $161 \mathrm{bp}$ \\
TGF $\beta$ Rv & AGGCTCCAAATGTAGGGGCAG & & \\
\hline
\end{tabular}

bp, base pair; GAPDH, glyceraldehyde-3-phosphate dehydrogenase; Fw, forward; Rv, reverse; SCCA, squamous cell carcinoma antigen; TGF $\beta$, transforming growth factor $\beta$. 
total tissue area (excluding airspaces) of the section (where $\mathrm{n}=$ the number of fields):

- FF ratio $=\Sigma_{\mathrm{n}} \mathrm{A}_{\mathrm{FF}} /$ total area $\times 100$

- IC ratio $=\Sigma_{\mathrm{n}} \mathrm{A}_{\mathrm{IC}} /$ total area $\times 100$

- Fibrosis ratio $=\Sigma_{n} A_{\text {FIB }} /$ total area $\times 100$.

For each patient, the ratios obtained from the analysed sections were then averaged and this value was correlated with all pathological and clinical parameters. In contrast with the authors recently supporting the value of quantitative analysis of $\mathrm{FF}^{20}$ we measured all parameters, including $\mathrm{FF}$, exclusively related to lung tissue (excluding air spaces) to normalise the effect of collapse or expansion of lung tissue during biopsy or tissue fixation.

\section{Immunohistochemical analysis}

All cases were immunoassayed with a novel polyclonal rabbit antibody anti-SCCA (Hepa-Ab, Xeptagen, Venice, Italy) and mouse monoclonal anti-TGF $\beta$ (NovoCastra, Newcastle, UK), as previously described. ${ }^{21}{ }^{22}$ All samples were processed using a sensitive avidin-streptavidin-peroxidase technique and stained with a mixture of 3,3-diaminobenzidine tetrahydrochloride and hydrogen peroxide. Parallel control slides were prepared either lacking primary antibody or lacking primary and secondary antibodies, or were stained with normal sera to control for background reactivity. Consecutive serial sections immunostained for SCCA and TGF $\beta$ were evaluated and the quantification was restricted to strongly stained metaplastic epithelial
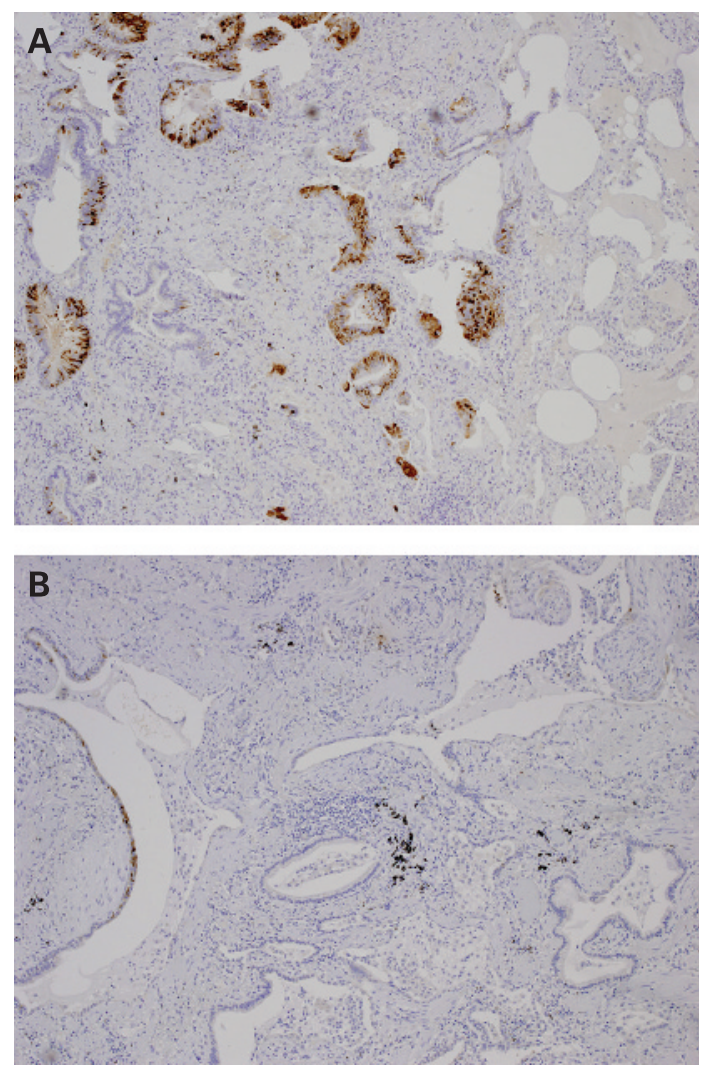

Figure 1 Immunohistochemistry for squamous cell carcinoma antigen (SCCA). (A) Usual interstitial pneumonia (UIP) case, female, 56 years old (SCCA mean value 44\%). Many metaplastic bronchiolar and cuboidal cells are strongly marked. Original magnification $\times 50$. (B) UIP case, male, 65 years old (SCCA mean value 10.5\%). A few metaplastic cuboidal and bronchiolar cells are moderately stained. Original magnification $\times 50$. cells (cuboidal, squamous, bronchiolar). A total of 500 metaplastic epithelial cells for each patient were counted in remodelled lung parenchyma (at least two sections) and the value was expressed as a percentage of positive cells/500 for each case. This value was correlated with all pathological and clinical values.

\section{Molecular analysis}

Manual tissue dissection

Manual tissue dissection of representative areas, positive for SCCA and TGF $\beta$, was performed in half of the cases (11 cases) in which metaplastic epithelial aggregates were easily dissected.
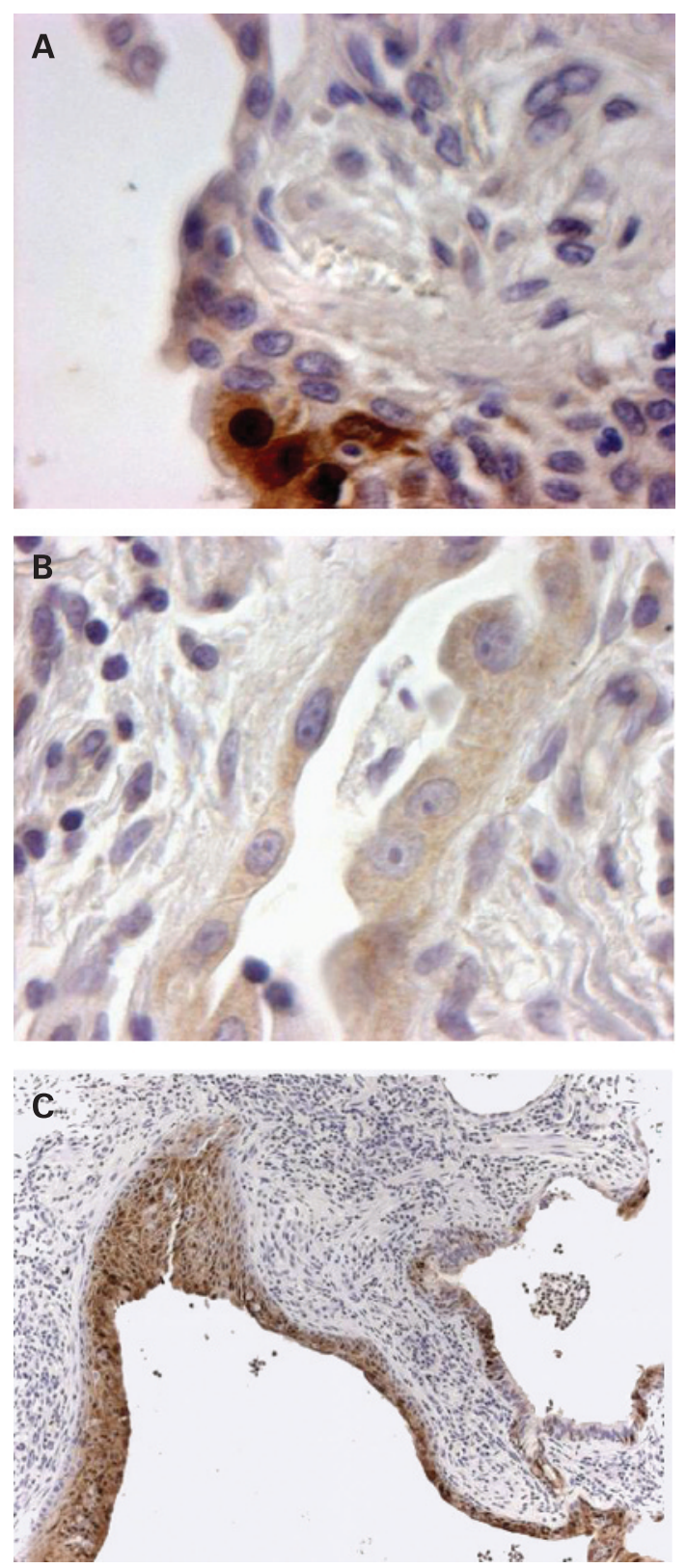

Figure 2 Immunohistochemistry for squamous cell carcinoma antigen (SCCA). Usual interstitial pneumonia (UIP) case, male, 66 years old (SCCA mean value 32\%). Strong nuclear and cytoplasmic staining of metaplastic bronchiolar cells $(A)$, weak cytoplasmic staining of metaplastic cuboidal cells $(B)$ (both original magnification $\times 350$ ) and strong cytoplasmic and nuclear staining of metaplastic squamous cells (C) (original magnification $\times 250$ ). 


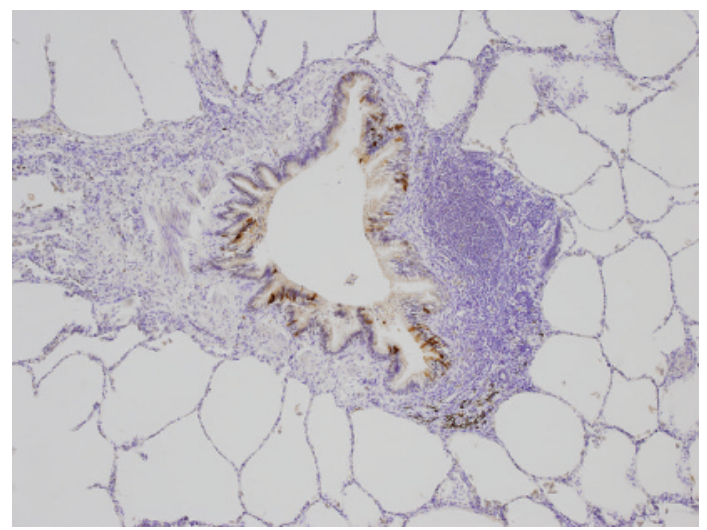

Figure 3 Immunohistochemistry for squamous cell carcinoma antigen (SCCA). Control case (non-implanted donor lung): positive staining is seen only in epithelial cells of the bronchial tract with well developed bronchial associated lymphoid tissue. Original magnification $\times 50$.

Briefly, five sequential $5 \mu \mathrm{m}$ sections from formalin fixed paraffin embedded blocks were placed on non-coated glass slides and coupled with SCCA and TGF $\beta$ immunostained tissue sections. The areas (at least $1 \mathrm{~mm}$ in diameter) carefully marked to easily compare the unstained levels were gently scraped with a sterile scalpel. The procured tissue fragments were then placed in a tube, deparaffinised and washed in xylene and alcohol before nucleic acid extraction. After this procedure, the remaining unselected tissue was stained with haematoxylineosin to verify the isolated tissue parts.

Areas of normal tissue (negative for SCCA and TGF $\beta$ immunoassaying) from the same paraffin block and from donor lung were also dissected and processed in the same way.

\section{RT-PCR of SCCA and TGF $\beta$}

Total RNA was extracted using the modified RNAzol method, as previously described. ${ }^{23}$ The RNA pellet was redissolved in $15 \mu \mathrm{l}$ of sterile diethylpirocarbonate treated water and incubated with $1 \mu \mathrm{l}$ of RNAse inhibitor (Applied Biosystems, Milan, Italy) and $20 \mathrm{U}$ of DNAse I (Sigma Aldrich, Milan, Italy) for $2 \mathrm{~h}$ at $37^{\circ} \mathrm{C}$ in a total volume of $20 \mu \mathrm{l}$. The oligonucleotides used to ascertain the quality of extracted RNA were complementary to the mRNA glyceraldehyde-3-phosphate dehydrogenase (GAPDH). The sequences of primers for GAPDH, SCCA and TGF $\beta$, annealing temperature condition and amplicon sizes are listed in table 2. At least $1 \mu \mathrm{g}$ of extracted total RNA was used for the first cDNA synthesis and conventional RT-PCR was used. The PCR mix was made up to a volume of $50 \mu \mathrm{l}$ using $1 \times$ PCR Buffer II, $1 \mathrm{mM} \mathrm{MgCl}_{2}$ solution, $200 \mu \mathrm{M}$ each of dATP, dCTP, dGTP and dUTP, $400 \mathrm{nM}$ of each primer and $1.25 \mathrm{U}$ of AmpliTaq Gold. After the initial denaturation at $95^{\circ} \mathrm{C}$ for 10 min, cDNA was amplified by 40 three step cycles ( $30 \mathrm{~s}$ at $95^{\circ} \mathrm{C}, 30 \mathrm{~s}$ at annealing temperature, $1 \mathrm{~min}$ at $72^{\circ} \mathrm{C}$ ). SCCA and TGF $\beta$ amplicons were both verified by a previously described gene sequencing protocol. ${ }^{21}$

\section{In vitro study}

\section{Cell culture}

Lung epithelial cell line A549 was obtained from the American Type Culture Collection (Manassas, Virginia, USA). Cells were maintained routinely in Dulbecco's modified Eagle's medium with $10 \%$ fetal bovine serum (Gibco, Milan, Italy) and
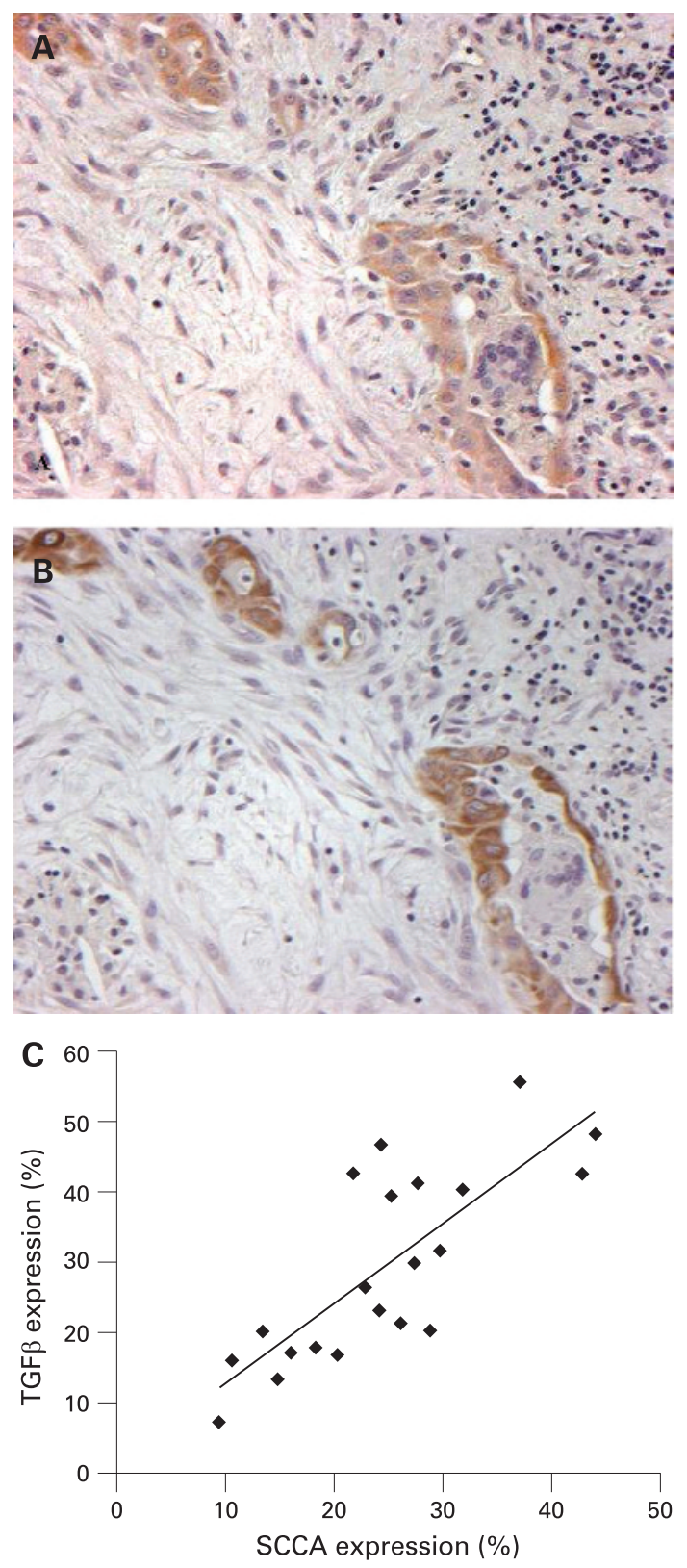

Figure 4 Immunohistochemistry for squamous cell carcinoma antigen (SCCA) and transforming growth factor $\beta$ (TGF $\beta)(A, B)$; SCCA and TGF $\beta$ correlation (C). Usual interstitial pneumonia (UIP) case, female, 61 years old. Note the strong staining in the same metaplastic cells of sequential serial sections. Original magnification $\times 300(A, B)$. Significant correlation between SCCA and TGF $\beta$ epithelial expression $(r=0.78$, $\mathrm{p}<0.0001$ ) (C).

supplemented with $100 \mathrm{mg} / \mathrm{ml}$ penicillin G (Gibco) and $100 \mathrm{mg} / \mathrm{ml}$ streptomycin sulfate (Gibco, Milan, Italy). Cells were then seeded in Dulbecco's modified Eagle's medium with $10 \%$ fetal bovine serum at a density of $0.3 \times 10^{6}$ cells per well in six well plates. All experiments were performed at $100 \%$ cell confluence. Time course analysis was carried out to assess the effect of SCCA on TGF $\beta$ expression. Cells were incubated with SCCA (Xeptagen, Venice, Italy) at increasing concentrations (range $1-1000 \mathrm{pg} / \mathrm{ml}$ ) and checked for TGF $\beta$ expression at $6 \mathrm{~h}$ intervals (range 6-48). Cells were collected and lysated immediately with RLT buffer (Qiagen, Milan, Italy) and $\beta$-mercaptoethanol 14.5 M 1\% (Sigma Aldrich, Milan, Italy). 

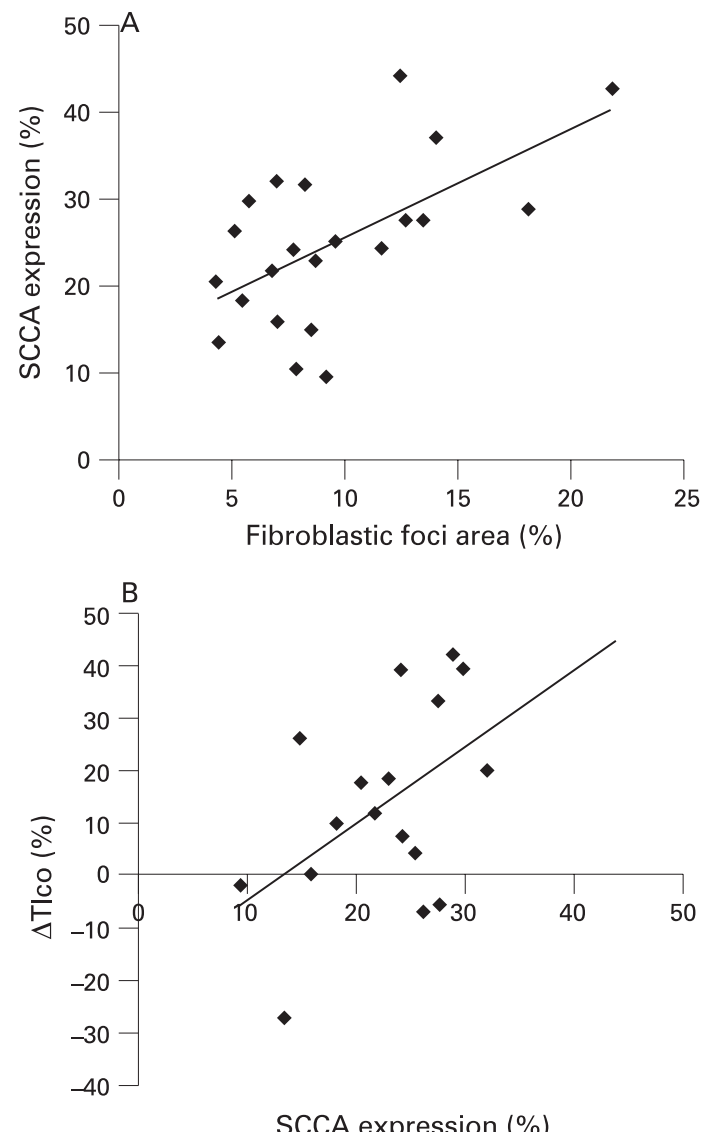

SCCA expression (\%)

Figure 5 Correlation between squamous cell carcinoma antigen (SCCA) and fibroblastic foci area (A) and SCCA and carbon monoxide transfer factor $\left(\triangle T I_{c 0}\right)(B)$. Direct correlation was observed between $A_{F F}$ and SCCA expression $(r=0.49, p=0.02)(A)$. A significant correlation was seen between SCCA expression and $\Delta \mathrm{TICO}(r=0.38, p=0.04)(B)$.

\section{Quantitative RT-PCR of TGF $\beta$}

RNA was extracted using RNeasy Mini Kit (Oiagen, Milan, Italy) according to the manufacturer's instructions. RNA was reverse transcribed using the Reverse Transcription System (Promega Corporation, Madison, Wisconsin, USA) according to the manufacturer's instructions. Real Time PCR was performed using a standard TaqMan PCR kit protocol on an Applied Biosystems 7000 Sequence Detection System (Applied Biosystems, Milan, Italy). The TaqMan PCR was carried out in a 96 well microtitre plate format (Applied Biosystems). The PCR mix was made up to a volume of $25 \mu \mathrm{l}$ using ready to use Universal Mastermix containing AmpliTaq DNA polymerase, uracil-N-glycosilase, dNTPs, $\mathrm{KCl}, \mathrm{MgCl}_{2}$ and $\mathrm{ROX}$ as passive reference, all in optimised concentrations. After uracil-Nglycosilase treatment at $50^{\circ} \mathrm{C}$ for 2 min and initial denaturation at $95^{\circ} \mathrm{C}$ for $10 \mathrm{~min}$, DNA was amplified by 40 two step cycles $\left(15 \mathrm{~s}\right.$ at $95^{\circ} \mathrm{C}, 1 \mathrm{~min}$ at $\left.57^{\circ} \mathrm{C}\right)$. All reactions were run in triplicate. Reactions and cycling were performed as recommended by the manufacturer. GAPDH was used as a reference gene for the adjustment of relative expression data. Naive cells collected at the same time were used as calibrators. All assays were performed in triplicate to ensure their reproducibility, and a negative control was included in each run. Primers and probes for GAPDH and TGF $\beta$ were commercially available (4333764 and 4327054; Applied Biosystems).

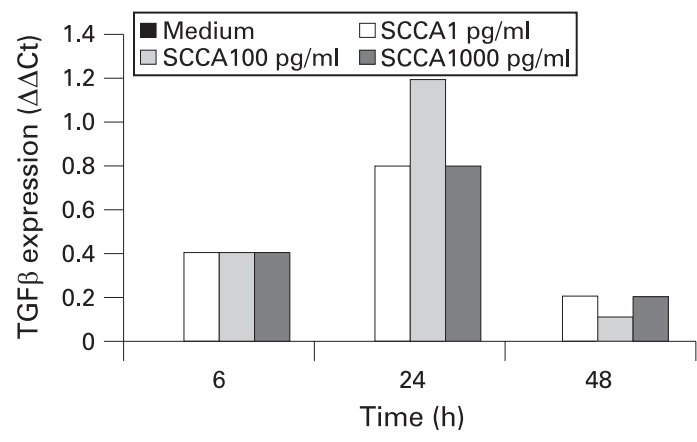

Figure 6 Effect of squamous cell carcinoma antigen (SCCA) at different time points in $\mathrm{A} 549$ cell line. At $24 \mathrm{~h}$, peak of transforming growth factor $\beta$ (TGF $\beta$ ) RNA transcription was observed and the highest induction was achieved with the $100 \mathrm{pg} / \mathrm{ml}$ SCCA concentration.

\section{Statistical analysis}

Normality of distribution for quantitative variables was assessed by means of Shapiro-Wilk statistics. Normally distributed quantitative variables are described as mean (SD), not normal quantitative variables are expressed as median, range and interquartile range (IOR), while categorical variables are presented as percentage distribution. To evaluate simple linear relationships between quantitative variables, Pearson's or Spearman's correlation coefficients were applied, as necessary. To evaluate the independent association of each factor with the dependent variable, the partial correlation coefficients were estimated.

In the analysis of the relationship between SCCA and scores, to allow greater set sizes, foci and inflammation scores were used to group IPF subjects. For foci, subjects were ranked: 1 (score 1-2), 2 (score 3-4) or 3 (score 5-6). For inflammation, the variable was dichotomised (score $\leqslant 1$, score $>1$ ). The mean value of SCCA of the different groups was compared by means of one way analysis of variance (ANOVA).

Statistical analysis was performed using SAS statistical software V.9.1 (SAS Institute, Carry, North Carolina, USA). A $p$ value $<0.05$ was considered statistically significant.

\section{RESULTS}

\section{Pathological findings and clinicopathological correlations}

Median histological score (range, IOR) was $3.0(1-6,2)$ and 1.0 $(1-3,1)$ for FF and for IC, respectively. Among all quantitative variables, only fibroblastic foci area $\left(\mathrm{A}_{\mathrm{FF}}\right)$ showed a distribution different from normality $(p=0.02)$. Median value for $A_{F F}$ was $8.4 \%(4.3-21.8 \%, 5.6 \%)$. Mean value for inflammation area $\left(\mathrm{A}_{\mathrm{IC}}\right)$ and fibrosis extension were $3.0(1.4) \%$ and $35.6(7.4) \%$, respectively (table 1 ). A direct correlation was observed between $A_{\text {FF }}$ and $A_{\text {FIB }}(r=0.56, p=0.007)$. A statistically significant correlation was observed only between $\mathrm{A}_{\mathrm{FIB}}$ and decline in Tlco at 9 months $(r=0.5156, p=0.0302)$.

\section{Immunohistochemical findings and correlations with morphological and clinical data}

SCCA was expressed in many metaplastic alveolar epithelial cells in all UIP cases, ranging from $9.4 \%$ to $44.0 \%$, and was normally distributed with a mean of 24.9 (9.3)\% (fig 1A, 1B). Immunoassaying was mainly detected in the cytoplasm but in a few cases cytoplasmic and nuclear staining was also observed (fig 2A). Cuboidal, flattened metaplastic epithelial cells showed a wide spectrum of staining from strong to weak SCCA 
Figure 7 Novel putative schema showing squamous cell carcinoma antigen (SCCA) pathway in usual interstitial pneumonia (UIP). SCCA could play a crucial role in the development of the disease: influencing epithelial proliferation (autocrine action) or promoting fibroblast proliferation/fibrosis through increased transforming growth factor $\beta$ (TGF $\beta$ ) secretion (paracrine action).

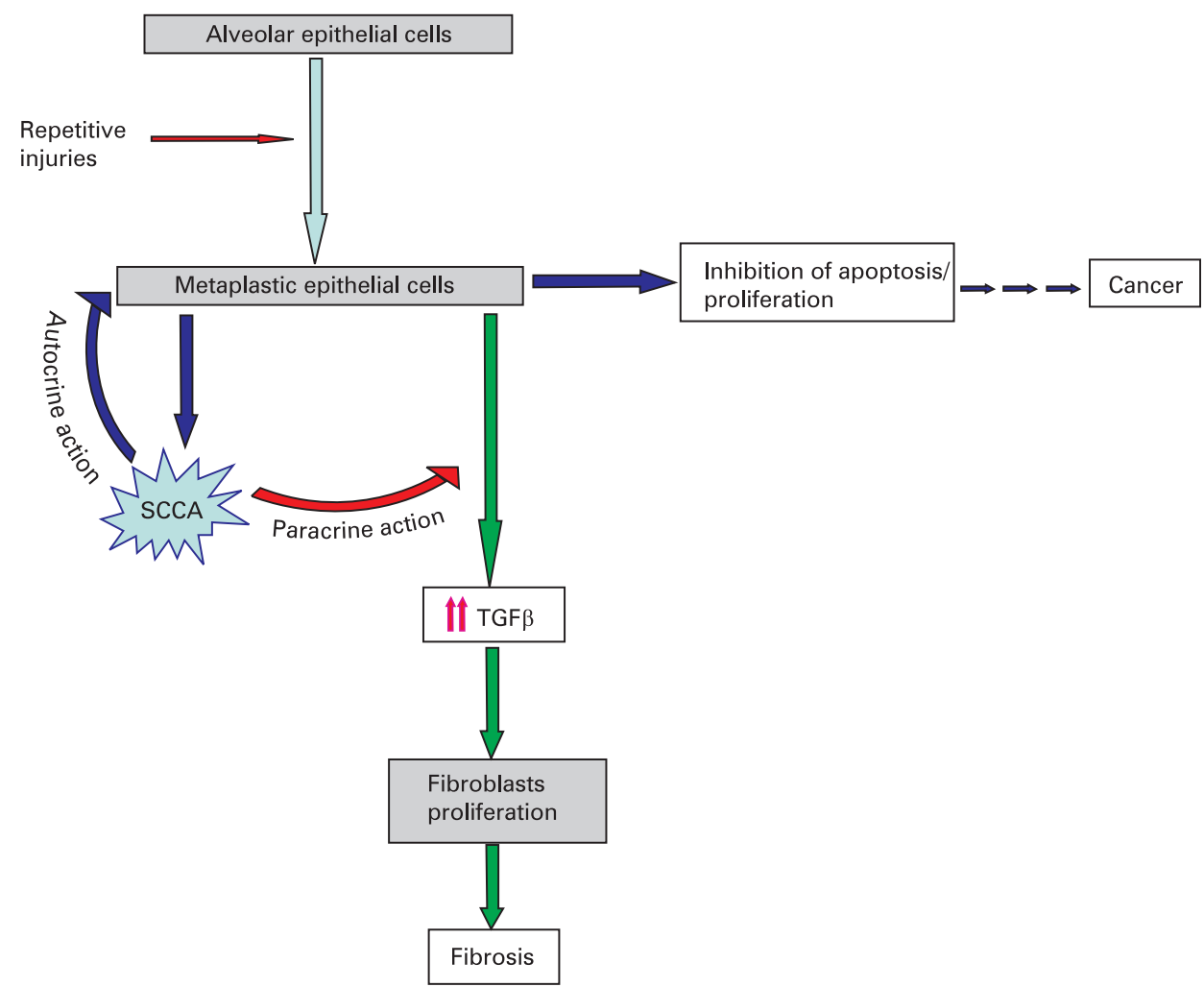

positivity (fig 2B) in contrast with squamous and bronchiolar metaplastic epithelial cells which frequently showed strong staining (fig 2C), more often well evident in or close to the honeycomb changes. Interstitial cells were negative for SCCA expression and a weak cytoplasmic staining was observed in some alveolar macrophages. Metaplastic epithelial SCCA positive staining was seen in only two ILDs different from IPF (non-specific interstitial pneumonia and desquamative interstitial pneumonia). The positivity was weak and mainly detected in cytoplasmic areas with a mean of $0.4(1.3) \%$ (from $0-5 \%$ ). The pseudostratified columnar epithelial cells lining the bronchi and bronchioles often exhibited strong cytoplasmic immunoreactivity for SCCA, thus representing a good internal immunostaining control. No alveolar cells were detected in normal lung tissue from donor subjects when only some bronchial cells were marked (fig 3).

In all UIP cases, TGF $\beta$, which often marked the same metaplastic epithelial cells positively stained for SCCA, showed a mean value of $29.8(13.5) \%(7.1-55.6 \%)$ and was significantly correlated with the expression values of SCCA $(r=0.78$, $\mathrm{p}<0.0001$ ) (fig 4A-C). TGF $\beta$ epithelial expression was occasionally seen in the control group (up to $2 \%$ ).

A direct correlation was observed between expression of SCCA and $A_{F F}(r=0.49, p=0.02)$ (fig $5 A$ ) and between TGF $\beta$ and $A_{F F}(r=0.44, p=0.04)$. Controlling for SCCA values, the correlation between TGF $\beta$ and $A_{F F}$ was no longer statistically significant (partial $r=0.10, p=0.67$ ). Mean SCCA values did not significantly differ both among foci score groups $(\mathrm{F}=0.65$, $\mathrm{p}=0.53)$ and inflammation score groups $(\mathrm{F}=0.36, \mathrm{p}=0.56)$. The only statistically significant relationship between SCCA values and lung clinical data variation during follow-up was observed with decreasing Tlco ( $\Delta$ Tlco) at 9 months $(r=0.38$, $\mathrm{p}=0.04)$ (fig 5B).

\section{SCCA and TGF $\beta$ mRNAs}

The expected 80 and $161 \mathrm{bp}$ PCR products of SCCA and TGF $\beta$, respectively, were detected from all pathological microdissected lung tissues, confirming epithelial transcription (data not shown).

\section{TGF $\beta$ mRNA expression in A549 cells incubated with SCCA}

Time course analysis of the effect of SCCA on TGF $\beta$ transcription in A549 cell lines is reported in fig 6. Peak induction of TGF $\beta$ transcription was achieved at $24 \mathrm{~h}$, with the highest activity of SCCA at the $100 \mathrm{pg} / \mathrm{ml}$ concentration.

\section{DISCUSSION}

The serpin superfamily includes inhibitors of a number of serine proteases with roles in a variety of cellular processes, including fibrinolysis, inflammation, cell migration, adhesion and proliferation. SCCA is transcribed from the tandemly repeated genes, SCCA1 and SCCA2, which have 98\% sequence identity at the nucleotide level and $92 \%$ identity at the amino acid level. ${ }^{16}$ The products of the two genes have different protease targets; SCCA1 inhibits papain-like cysteine proteases (cathepsin S, I and $\mathrm{K})^{24}$ and SCCA 2 chymotrypsin-like serine proteases (eg, catepsin G and mast cell chymase). ${ }^{25}$ While some studies have reported increased SCCA expression (mainly in blood serum) in many tumours, particularly those with squamous cell differentiation, ${ }^{26}{ }^{27}$ and in preneoplastic bronchial lesions, ${ }^{28}$ little is known about the behaviour of this serpin in a non-neoplastic clinical setting.

In the present study, we have demonstrated for the first time overexpression of SCCA in lung tissue of IPF/UIP patients compared with other forms of ILDs and normal lungs. In IPF, SCCA was abnormally secreted by metaplastic epithelial cells other than bronchial cells where it is normally expressed. The 
normal presence of SCCA at this level, as was demonstrated in our control cases, could have a protective function against inflammatory cells and microorganisms because SCCA 1 and 2 are inhibitors of serine and cysteine proteinase. Previous studies have reported that SCCA can have an important influence on epithelial growth through inhibition of different apoptotic pathways. ${ }^{29} 30$ The marked positivity of SCCA in the honeycomb area suggests that this site is more frequently subjected to injury favouring epithelial proliferation, which could be highly unstable when recurrent injuries occur.

Several years ago, Meyer and Liebow already showed atypical epithelial lesions in honeycombing pulmonary areas in IPF, raising the possibility that atypical epithelial lesions in IPF might be precancerous lesions. ${ }^{5}$ Squamous metaplasia in our samples showed frequent and strong staining with SCCA. It is probable that this epithelial setting represents a regeneration epithelial site of high instability at risk of neoplastic transformation. A recent in vitro study has shown overexpression of SCCA in squamous metaplastic tracheobronchial cell lines, increasing with tumour progression. ${ }^{31}$ Squamous-type carcinoma has in fact been detected in IPF/UIP lungs more frequently than in other tumour types. ${ }^{9}$

The nuclear/cytoplasmic concomitant SCCA immunoreactivity observed in our samples is difficult to interpret. Other works have recently described nuclear-cytoplasmic positive staining of serpin (maspin and SCCA) in non-small cell lung carcinoma. In this work, the nuclear maspin positivity was correlated with a better survival than with cytoplasmic staining. ${ }^{32}$ The synthesis of SCCA is thought to be essentially in the cytoplasm. However, when it is overexpressed, nuclear import might occur, thus playing a role in influencing the transcription of different growth factor genes, such as TGF $\beta$. Significant overexpression of TGF $\beta$ was detected in our cases and interestingly it was significantly correlated with SCCA expression. The increased TGF $\beta$ transcription by SCCA observed in A549 pneumocytes confirms clinical observations detected in patients with IPF/UIP. Unfortunately, it is nearly impossible to work with primary alveolar epithelial cells, and we had to resort to the epithelial cell line A549, used in numerous studies of IPF. There are different sources of TGF $\beta$ in IPF/UIP lungs, and active TGF $\beta$ transcription was detected in many metaplastic epithelial cells obtained by microdissection of our samples. Active epithelial secretion of this profibrogenetic cytokine was first reported by Corrin and colleagues ${ }^{33}$ and subsequently by other authors, ${ }^{34}$ thus underlining the important contributing role of epithelial cells in the development/progression of the fibrotic process in IPF/UIP.

The significant correlation between expression of SCCA and extension of FF and fibrosis could be mediated through the increased transcription of the fibrotic cytokine TGF $\beta$, confirming the role of this cytokine as one of the most important inducers of fibrotic processes in IPF/UIP. Our morphological evaluation confirms the prognostic significance of the quantitative measure of fibrosis and FF (instead of semiquantitative evaluation) in that these are the only pathological findings significantly correlated with Tlco decline and SCCA expression, as reported above. The significant correlation of SCCA with Tlco decline observed in our patients after 9 months of followup could be related to progressive fibrosis favoured by increased TGF $\beta$ transcription. However, it remains to be established if alveolar epithelial dysfunction could represent an important contributing factor for progressive impaired gas exchange.

Although our findings need to be confirmed in larger case series, we consider SCCA an important molecular target in the disease as it could orchestrate two of the most peculiar aspects of the disease: this serpin could act in an autocrine way favouring epithelial proliferation and metaplastic dysplastic transformation while in a paracrine way it could influence myofibroblast proliferation and collagen synthesis through increased TGF $\beta$ transcription (fig 7). Monitoring of tissue SCCA expression during the clinical course of IPF/UIP could be useful for more precise patient stratification. It could be advisable to use a non-invasive approach such as innovative serological analysis of SCCA-immunoglobulin M complex, a more sensitive and appropriate technique ${ }^{35}$ that those used previously.

A final issue that needs to be taken into account is the possible role of SCCA in the development of lung cancer in IPF/ UIP. Analysis of this specific aspect could be proven in studies of tissue samples of cancer in UIP and by using specific experimental models, such as those involving transgenic SCCA animal models recently engineered in our laboratory.

Acknowledgements: The authors thank Luca Braghetto and Laura Vignato for their excellent technical assistance and Judith Wilson for English revision.

Funding: This study was supported by the Italian Ministry of Instruction, University and Research.

Competing interests: None

Ethics approval: The study was approved by the Institutional Ethics Committee.

\section{REFERENCES}

1. Crystal RG, Bitterman PB, Mossman B, et al. Future research directions in idiopathic pulmonary fibrosis: summary of a National Heart, Lung, and Blood Institute working group. Am J Respir Crit Care Med 2002;166:236-46.

2. Selman M, King TE, Pardo A, American Thoracic Society, European Respiratory Society, American College of Chest Physicians. Idiopathic pulmonary fibrosis: prevailing and evolving hypotheses about its pathogenesis and implications for therapy. Ann Intern Med 2001;134:136-51.

3. Calabrese F, Giacometti C, Rea F, et al. Idiopathic interstitial pneumonias: Primum movens: epithelial, endothelial or whatever. Sarcoidosis Vasc Diffuse Lung Dis 2005:22:S15-23.

4. Nicholson AG, Fulford LG, Colby TV, et al. The relationship between individual histologic features and disease progression in idiopathic pulmonary fibrosis. Am J Respir Crit Care Med 2002;166:173-7.

5. Meyer EC, Liebow AA. Relationship of interstitial pneumonia honeycombing and atypical epithelial proliferation to cancer of the lung. Cancer 1965;18:322-51.

6. Hironaka M, Fukayama M. Pulmonary fibrosis and lung carcinoma: a comparative study of metaplastic epithelia in honeycombed areas of usual interstitial pneumonia with or without lung carcinoma. Pathol Int 1999;49:1060-6.

7. Hubbard R, Venn A, Lewis S, et al. Lung cancer and cryptogenic fibrosing alveolitis. A population-based cohort study. Am J Respir Crit Care Med 2000;161:5-8.

8. Park J, Kim DS, Shim TS, et al. Lung cancer in patients with idiopathic pulmonary fibrosis. Eur Respir J 2001;17:1216-19.

9. Aubry MC, Myers JL, Douglas WW, et al. Primary pulmonary carcinoma in patients with idiopathic pulmonary fibrosis. Mayo Clin Proc 2002;77:763-70.

10. Ma Y, Seneviratne CK, Koss M. Idiopathic pulmonary fibrosis and malignancy. Curr Opin Pulm Med 2001;7:278-82.

11. Takahashi T, Munakata $M$, Ohtsuka $Y$, et al. Expression and alteration of ras and p53 proteins in patients with lung carcinoma accompanied by idiopathic pulmonary fibrosis. Cancer 2002;95:624-33.

12. Kuwano K, Kunitake R, Kawasaki M, et al. P21Waf1/Cip1/Sdi1 and p53 expression in association with DNA strand breaks in idiopathic pulmonary fibrosis. Am J Respir Crit Care Med 1996;154:477-83.

13. Chilosi M, Poletti V, Murer B, et al. Abnormal re-epithelialization and lung remodeling in idiopathic pulmonary fibrosis: the role of deltaN-p63. Lab Invest 2002;82:1335-45.

14. Terasaki Y, Akuta T, Terasaki M, et al. Guanine nitration in idiopathic pulmonary fibrosis and its implication for carcinogenesis. Am J Respir Crit Care Med 2006;174:665-73

15. Kato H. Expression and function of squamous cell carcinoma antigen. Anticancer Res 1996;16:2149-53.

16. Schneider SS, Schick C, Fish KE, et al. A serine proteinase inhibitor locus at $18 \mathrm{q} 21.3$ contains a tandem duplication of the human squamous cell carcinoma antigen gene. Proc Natl Acad Sci U S A 1995;92:3147-51

17. Suminami Y, Nagashima $S$, Murakami $A$, et al. Suppression of a squamous cell carcinoma (SCC)-related serpin, SCC antigen, inhibits tumor growth with increased intratumor infiltration of natural killer cells. Cancer Res 2001;61:1776-80.

18. American Thoracic Society, European Respiratory Society. American Thoracic Society/European Respiratory Society International Multidisciplinary Consensus 
Classification of the Idiopathic Interstitial Pneumonias. This joint statement of the American Thoracic Society (ATS), and the European Respiratory Society (ERS) was adopted by the ATS board of directors, June 2001 and by the ERS Executive Committee, June 2001. Am J Respir Crit Care Med 2002:165:277-304.

19. Dona M, Dell'Aica I, Pezzato E, et al. Hyperforin inhibits cancer invasion and metastasis. Cancer Res 2004;64:6225-32.

20. Enomoto $\mathbf{N}$, Suda T, Kato M, et al. Quantitative analysis of fibroblastic foci in usual interstitial pneumonia. Chest 2006;130:22-9.

21. Pontisso P, Calabrese F, Benvegnu L, et al. Overexpression of squamous cell carcinoma antigen variants in hepatocellular carcinoma. Br J Cancer 2004:90:833-7.

22. Beghe B, Bazzan E, Baraldo S, et al. Transforming growth factor-beta type II receptor in pulmonary arteries of patients with very severe COPD. Eur Respir J 2006:28:556-62.

23. Chomczynski $\mathbf{P}$, Sacchi N. Single-step method of RNA isolation by guanidium thiocyanate-phenol-chloroform extraction. Anal Biochem 1987;162:156-9.

24. Schick C, Pemberton PA, Shi GP, et al. Cross-class inhibition of the cysteine proteinases cathepsins $K, L$, and $S$ by the serpin squamous cell carcinoma antigen 1 : a kinetic analysis. Biochemistry 1998;37:5258-66.

25. Schick C, Kamachi Y, Bartuski AJ, et al. Squamous cell carcinoma antigen 2 is a novel serpin that inhibits the chymotrypsin-like proteinases cathepsin $\mathrm{G}$ and mast cell chymase. J Biol Chem 1997;272:1849-55.

26. Body JJ, Sculier JP, Raymakers N, et al. Evaluation of squamous cell carcinoma antigen as a new marker for lung cancer. Cancer 1990;65:1552-6.

27. Vassilakopoulos T, Troupis T, Sotiropoulou C, et al. Diagnostic and prognostic significance of squamous cell carcinoma antigen in non-small cell lung cancer. Lung Cancer 2001;32:137-44.
28. Smith SL, Watson SG, Ratschiller D, et al. Maspin-the most commonly-expressed gene of the 18q21.3 serpin cluster in lung cancer-is strongly expressed in preneoplastic bronchial lesions. Oncogene 2003;22:8677-87.

29. Murakami A, Suminami $Y$, Hirakawa $H$, et al. Squamous cell carcinoma antigen suppresses radiation-induced cell death. Br J Cancer 2001;84:851-8.

30. Suminami Y, Kishi F, Sekiguchi K, et al. Squamous cell carcinoma antigen is a new member of the serine protease inhibitors. Biochem Biophys Res Commun 1991;181:51-8.

31. Lonardo F, Li X, Siddiq F, et al. Maspin nuclear localization is linked to favorable morphological features in pulmonary adenocarcinoma. Lung Cance 2006; 51:31-9.

32. Kim SW, Cheon K, Kim CH, et al. Proteomics-based identification of proteins secreted in apical surface fluid of squamous metaplastic human tracheobronchia epithelial cells cultured by three-dimensional organotypic air-liquid interface method. Cancer Res 2007:67:6565-73.

33. Corrin B, Butcher D, McAnulty BJ, et al. Immunohistochemical localization of transforming growth factor-beta 1 in the lungs of patients with systemic sclerosis, cryptogenic fibrosing alveolitis and other lung disorders. Histopathology 1994;24:145-50

34. Khalil N, O'Connor RN, Flanders KC, et al. TGF-beta 1, but not TGF-beta 2 or TGFbeta 3 , is differentially present in epithelial cells of advanced pulmonary fibrosis: an immunohistochemical study. Am J Respir Cell Mol Biol 1996;14:131-8.

35. Beneduce L, Castaldi F, Marino $M$, et al. Squamous cell carcinoma antigenimmunoglobulin $\mathrm{M}$ complexes as novel biomarkers for hepatocellular carcinoma. Cancer 2005;103:2558-65.

\section{Pulmonary puzzle}

\section{Acute respiratory distress in a patient using non-invasive ventilation}

\section{CLINICAL PRESENTATION}

A 49-year-old man with advanced motor neurone disease was admitted for initiation of non-invasive ventilation (NIV) for established daytime hypercapnia and symptoms of nocturnal hypoventilation. It had also been noted that when sleeping he was snoring heavily. He had initially tolerated NIV well during the day, but during his second night he was noted to be in respiratory distress with worsening arterial blood gases. He refused to use his ventilator any further. Communication was extremely limited due to severe bulbar symptoms, but the patient indicated facial discomfort. The chest radiograph was normal and a lateral facial radiograph is shown in fig 1.

\section{QUESTION}

What is the abnormality and the likely explanation for it?

See page 844

This case was submitted by:

\section{K S Bonnici, W D-C Man, M I Polkey}

Sleep and Ventilation Unit, Royal Brompton Hospital, London, UK

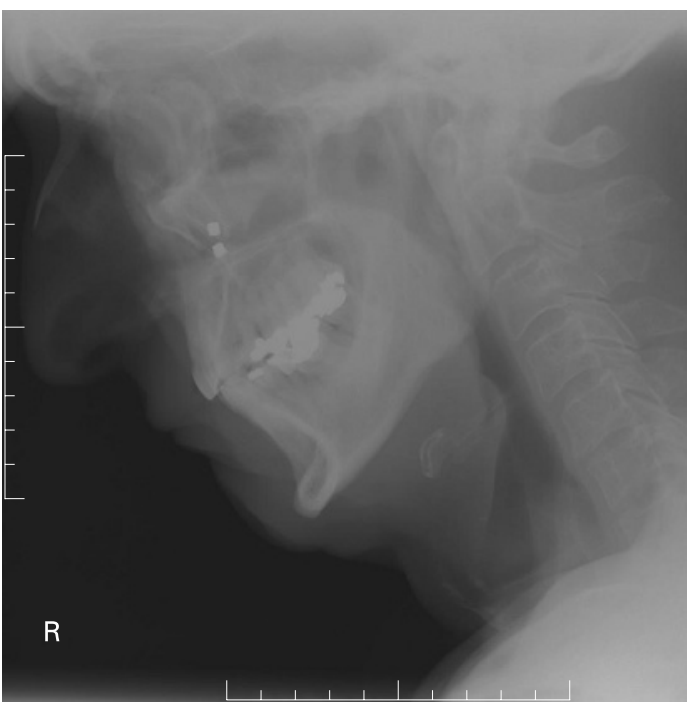

Figure 1 Lateral facial radiograph.

Correspondence to: Dr K S Bonnici, Royal Brompton Hospital, London, UK k.bonnici@rbht.nhs.uk

Competing interests: None.

Thorax 2008;63:802. doi:10.1136/thx.2007.093062 